\title{
Filtering on Nonlinear Time-Delay Stochastic Systems*
}

\author{
Zidong Wang ${ }^{\dagger}$ and Daniel W. C. $\mathrm{Ho}^{\ddagger}$
}

\begin{abstract}
In this paper, we address the filtering problem for a general class of nonlinear timedelay stochastic systems. The purpose of this problem is to design a full-order filter such that the dynamics of the estimation error is guaranteed to be stochastically exponentially ultimately bounded in the mean square. Both filter analysis and synthesis problems are considered. Sufficient conditions are proposed for the existence of desired exponential filters, which are expressed in terms of the solutions to algebraic Riccati inequalities involving scalar parameters. The explicit characterization of the desired filters is also derived. A simulation example is given to illustrate the design procedures and performances of the proposed method.
\end{abstract}

Key Words - Nonlinear filtering; Nonlinear systems; Stochastic exponential ultimate boundedness; Time-delay systems; Algebraic Riccati inequalities

\section{Introduction}

Nonlinear filtering for stochastic systems has been an active area of research over the past three decades. Since the time evolution of the probability density of the state vector conditional on the measurements cannot be directly calculated in most cases [1], various approximations have been developed in the literature, such as extended Kalman filters [5], and statistically linearized filters [10], see [2] for a survey. Other techniques include the bound-optimal filters [14], exponentially bounded filters [15, 21], approximations by Markov chains [19], minimum variance filters [20], approximation of the Kushner equation [6], to name just a few. It should be pointed out that, unlike the linear case, in most literature mentioned above, the solution to the nonlinear filtering problem has been given as a nonexplicit representation.

On the other hand, it has now been well known that the delayed state is very often the cause for instability and poor performance of systems. Compared to the robust $H_{\infty}$ control problems for linear systems with certain types of time-delays (see [12]), the "dual" filter/observer design problems have received much less attention although they are important in control design and signal processing applications. In [16, 17], the robust observer design problem has been considered for linear deterministic time-delay systems. In the

\footnotetext{
*accepted to Automatica with paper number TS-469 (global number 397). This paper was not presented at any IFAC meeting.

${ }^{\dagger} \mathrm{Z}$. Wang is the Department of Information Systems and Computing, Brunel University, Uxbridge, Middlesex, UB8 3PH, U.K. Email: Zidong.Wang@brunel.ac.uk, Fax: ++44/1895 251686.

${ }^{\ddagger}$ D. W. C. Ho is with Department of Mathematics, City University of Hong Kong, 83 Tat Chee Avenue, Kowloon, Hong Kong.
} 
stochastic framework, the Kalman filter design problem has been tackled in $[3,11]$ for linear continuous- and discrete-time cases, respectively. So far, to the best of the authors' knowledge, the issue of filtering on general nonlinear time-delay systems has not been fully investigated and remains to be important and challenging.

In this paper, we consider the filtering problem for a general class of nonlinear timedelay stochastic systems, where the nonlinearities are assumed to have the similar form as in [21]. We aim at deriving the conditions for a full-order filter such that the dynamics of the estimation error is guaranteed to be stochastically exponentially ultimately bounded in the mean square. Both filter analysis and synthesis issues are addressed. It is shown that the existence of desired exponential filters can be guaranteed if some algebraic Riccati inequalities have positive definite solutions. In particular, the explicit expression of the desired filters is given in terms of some free parameters.

Notation. $\mathbb{R}^{n}$ and $\mathbb{R}^{n \times m}$ denote, respectively, the $n$ dimensional Euclidean space and the set of all $n \times m$ real matrices. The superscript " $T$ " denotes the transpose and the notation $X \geq Y$ (respectively, $X>Y$ ) where $X$ and $Y$ are symmetric matrices, means that $X-Y$ is positive semi-definite (respectively, positive definite). We let $\tau>0$ and $C\left([-\tau, 0] ; \mathbb{R}^{n}\right)$ denote the family of continuous functions $\varphi$ from $[-\tau, 0]$ to $\mathbb{R}^{n}$ with the norm $\|\varphi\|=$ $\sup _{-\tau \leq \theta \leq 0}|\varphi(\theta)|$, where $|\cdot|$ is the Euclidean norm in $\mathbb{R}^{n}$. If $A$ is a matrix, denote by $\|A\|$ its operator norm. $\lambda_{\max }(\cdot)$ (respectively, $\left.\lambda_{\min }(\cdot)\right)$ means the largest (respectively, smallest) eigenvalue of $A \cdot \operatorname{tr}(A)$ is the trace of the matrix $A$. Moreover, let $\left(\Omega, \mathcal{F},\left\{\mathcal{F}_{t}\right\}_{t \geq 0}, P\right)$ be a complete probability space with a filtration $\left\{\mathcal{F}_{t}\right\}_{t \geq 0}$ satisfying the usual conditions (i.e., the filtration contains all $P$-null sets and is right continuous). Denote by $L_{\mathcal{F}_{0}}^{p}\left([-\tau, 0] ; \mathbb{R}^{n}\right)$ the family of all $\mathcal{F}_{0}$-measurable $C\left([-\tau, 0] ; \mathbb{R}^{n}\right)$-valued random variables $\xi=\{\xi(\theta):-\tau \leq \theta \leq$ $0\}$ such that $\sup _{-\tau \leq \theta \leq 0} \mathbb{E}|\xi(\theta)|^{p}<\infty$ where $\mathbb{E}\{\cdot\}$ stands for the mathematical expectation operator with respect to the given probability measure $P$.

\section{Problem formulation and assumptions}

Consider the following nonlinear continuous-time state delayed stochastic system in a fixed complete probability space $\left(\Omega, \mathcal{F},\left\{\mathcal{F}_{t}\right\}_{t \geq 0}, P\right)$ :

$$
\begin{aligned}
& \dot{x}(t)=f(x(t), u(t))+g(x(t-\tau))+E_{1} w(t), \\
& x(t)=\varphi(t), \quad t \in[-\tau, 0],
\end{aligned}
$$

together with the measurement equation

$$
y(t)=h(x(t))+E_{2} w(t)
$$

where $x(t) \in \mathbb{R}^{n}$ is the state, $u(t) \in \mathbb{R}^{m}$ is the deterministic input, $y(t) \in \mathbb{R}^{p}$ is the measurement output, and $f(\cdot, \cdot) \in \mathbb{R}^{n}, g(\cdot) \in \mathbb{R}^{n}, h(\cdot) \in \mathbb{R}^{p}$ are nonlinear vector functions. $\tau$ denotes the known state delay, $\varphi(t)$ is a continuous vector valued initial function. Here, $w(t)$ is a zero mean Gaussian white noise process with covariance $I>0$. The initial state $x(0)$ has the mean $\bar{x}(0)$ and covariance $P(0)$, and is uncorrelated with $w(t) . E_{1}, E_{2}$ are known constant matrices with appropriate dimensions.

Assumption 2.1 The nonlinear vector functions $f(\cdot, \cdot), g(\cdot), h(\cdot)$ are assumed to satisfy 


$$
\begin{gathered}
f(0,0)=0, g(0)=0, h(0)=0 \text { and } \\
\qquad\left|f(x(t)+\sigma, u(t)+\delta)-f(x(t), u(t))-\left[\begin{array}{ll}
A & B
\end{array}\right]\left[\begin{array}{c}
\sigma \\
\delta
\end{array}\right]\right| \leq a_{11}\left|\left[\begin{array}{c}
\sigma \\
\delta
\end{array}\right]\right|, \\
\left|g(x(t-\tau)+\sigma)-g(x(t-\tau))-A_{d} \sigma\right| \leq a_{22}|\sigma|, \\
|h(x(t)+\sigma)-h(x(t))-C \sigma| \leq a_{33}|\sigma|
\end{gathered}
$$

where $A \in \mathbb{R}^{n \times n}, B \in \mathbb{R}^{n \times m}, A_{d} \in \mathbb{R}^{n \times n}, C \in \mathbb{R}^{p \times n}$ are known constant matrices, $\sigma \in \mathbb{R}^{n}$, $\delta \in \mathbb{R}^{m}$ are vectors, $a_{11}, a_{22}$, and $a_{33}$ are known positive constants.

Remark 2.1 Similar to [21], the nonlinear descriptions (2.4)-(2.6) quantify the maximum possible derivations from a linear model with $\left(A, B, A_{d}, C\right)$ as its system parameter matrices, and are more general than those of $[14,15]$.

In this paper, the full-order filter under consideration is of the form

$$
\dot{\hat{x}}(t)=f(\hat{x}(t), u(t))+g(\hat{x}(t-\tau))+K[y(t)-h(\hat{x}(t))]
$$

where $\hat{x}$ is the state estimate and the constant matrix $K$ is the filter gain to be designed. Let the error state be $e(t)=x(t)-\hat{x}(t)$, then it follows from (2.1)-(2.3) and (2.7) that

$$
\begin{aligned}
\dot{e}(t)= & f(x(t), u(t))-f(\hat{x}(t), u(t))+g(x(t-\tau))-g(\hat{x}(t-\tau)) \\
& -K[h(x(t))-h(\hat{x}(t))]+\left(E_{1}-K E_{2}\right) w(t) .
\end{aligned}
$$

For notational convenience, we give the following definitions:

$$
\begin{aligned}
l(t) & =f(x(t), u(t))-f(\hat{x}(t), u(t))-A e(t), \\
m(t-\tau) & =g(x(t-\tau))-g(\hat{x}(t-\tau))-A_{d} e(t-\tau), \\
n(t) & =h(x(t))-h(\hat{x}(t))-C e(t),
\end{aligned}
$$

and then obtain from (2.8) that

$$
\begin{aligned}
\dot{e}(t)= & (A-K C) e(t)+A_{d} e(t-\tau)+l(t)+m(t-\tau) \\
& -K n(t)+\left(E_{1}-K E_{2}\right) w(t) .
\end{aligned}
$$

Now, let $e(t ; \xi)$ denote the state trajectory from the initial data $e(\theta)=\xi(\theta)$ on $-\tau \leq \theta \leq 0$ in $L_{\mathcal{F}_{0}}^{2}\left([-\tau, 0] ; \mathbb{R}^{n}\right)$. We introduce the following concept.

Definition $2.1[21,22]$ For every $\xi \in L_{\mathcal{F}_{0}}^{2}\left([-\tau, 0] ; \mathbb{R}^{n}\right)$, the dynamics of the estimation error (i.e., the solution of the system (2.12)) is exponentially ultimately bounded in the mean square if there exist constants $\alpha>0, \beta>0, \gamma>0$ such that

$$
\mathbb{E}|e(t ; \xi)|^{2} \leq \alpha e^{-\beta t} \sup _{-\tau \leq \theta \leq 0} \mathbb{E}|\xi(\theta)|^{2}+\gamma
$$

Moreover, the filter (2.7) is said to be exponential if, for every $\xi \in L_{\mathcal{F}_{0}}^{2}\left([-\tau, 0] ; \mathbb{R}^{n}\right)$, the system (2.12) is exponentially ultimately bounded in the mean square.

The objective of this paper is to design an exponential filter for the nonlinear time-delay system (2.1)-(2.3). More specifically, we are interested in designing the filter parameter $K$ such that the dynamics of the estimation error (i.e., the solution of the system (2.12)) is guaranteed to be stochastically exponentially ultimately bounded in the mean square. 


\section{Main results and proofs}

In this section, the filter analysis problem will be considered firstly. For a given filter structure, we shall establish the conditions under which the estimation error is stochastically exponentially ultimately bounded in the mean square. The filter design problem will then be taken into account. We shall derive the explicit expression of the expected filter parameter in terms of the positive definite solution to a Riccati-like matrix inequality.

\subsection{Filter analysis}

Theorem 3.1 Let the filter parameter $K$ be given. If there exist positive scalars $\varepsilon_{1}, \varepsilon_{2}, \varepsilon_{3}, \varepsilon_{4}$ such that the following matrix inequality

$$
\begin{aligned}
(A-K C)^{T} P+ & P(A-K C)+P\left[\left(\varepsilon_{1}+\varepsilon_{2}+\varepsilon_{3}\right) I+\varepsilon_{4} K K^{T}\right] P \\
& +\left(\varepsilon_{2}^{-1} a_{11}^{2}+\varepsilon_{4}^{-1} a_{33}^{2}\right) I+Q<0
\end{aligned}
$$

where

$$
Q:=\varepsilon_{1}^{-1} A_{d}^{T} A_{d}+\varepsilon_{3}^{-1} a_{22}^{2} I
$$

has a positive definite solution $P>0$, then the system (2.12) is exponentially ultimately bounded in the mean square.

Proof: $\quad$ Fix $\xi \in L_{\mathcal{F}_{0}}^{2}\left([-\tau, 0] ; \mathbb{R}^{n}\right)$ arbitrarily and write $e(t ; \xi)=e(t)$. For $(e(t), t) \in$ $\mathbb{R}^{n} \times \mathbb{R}_{+}$, we define the Lyapunov function candidate

$$
V(e(t), t)=e^{T}(t) P e(t)+\int_{t-\tau}^{t} e^{T}(s) Q e(s) d s
$$

where $P>0$ is the solution to the matrix inequality (3.1) and $Q>0$ is defined in (3.2).

By Itô's formula (see, e.g., [9]), the stochastic derivative of $V$ along a given trajectory (2.12) is obtained as

$$
\begin{aligned}
\frac{d}{d t} V(e(t), t)= & e^{T}(t)\left[(A-K C)^{T} P+P(A-K C)+Q\right] e(t) \\
& +e^{T}(t) P A_{d} e(t-\tau)+e(t-\tau)^{T} A_{d}^{T} P e(t) \\
& +e^{T}(t) P[l(t)+m(t-\tau)-K n(t)]+[l(t)+m(t-\tau)-K n(t)]^{T} P e(t) \\
& -e^{T}(t-\tau) Q e(t-\tau)+\operatorname{tr}\left[\left(E_{1}-K E_{2}\right)\left(E_{1}-K E_{2}\right)^{T} P\right] \\
& +2 e^{T} P\left(E_{1}-K E_{2}\right) w(t) .
\end{aligned}
$$

Let $\varepsilon_{1}, \varepsilon_{2}, \varepsilon_{3}, \varepsilon_{4}$ be positive scalars. Then the matrix inequality

$$
\left[\varepsilon_{1}^{1 / 2} e^{T}(t) P-\varepsilon_{1}^{-1 / 2} e^{T}(t-\tau) A_{d}^{T}\right]\left[\varepsilon_{1}^{1 / 2} e^{T}(t) P-\varepsilon_{1}^{-1 / 2} e^{T}(t-\tau) A_{d}^{T}\right]^{T} \geq 0
$$

yields

$$
\begin{aligned}
& e^{T}(t) P A_{d} e(t-\tau)+e^{T}(t-\tau) A_{d}^{T} P e(t) \\
& \leq \varepsilon_{1} e^{T}(t) P^{2} e(t)+\varepsilon_{1}^{-1} e^{T}(t-\tau) A_{d}^{T} A_{d} e(t-\tau) .
\end{aligned}
$$


Noticing the Assumption 2.1 and (2.9)-(2.11), we have

$$
\begin{gathered}
l^{T}(t) l(t)=|f(x(t), u(t))-f(\hat{x}(t), u(t))-A e(t)|^{2} \leq a_{11}^{2}|e(t)|^{2}=a_{11}^{2} e^{T}(t) e(t), \\
m^{T}(t-\tau) m(t-\tau)=\left|g(x(t-\tau))-g(\hat{x}(t-\tau))-A_{d} e(t-\tau)\right|^{2} \\
\left.\leq a_{22}^{2} \mid e(t-\tau)\right)\left.\right|^{2}=a_{22}^{2} e^{T}(t-\tau) e(t-\tau) \\
n^{T}(t) n(t)=|h(x(t))-h(\hat{x}(t))-C e(t)|^{2} \leq a_{33}^{2}|e(t)|^{2}=a_{33}^{2} e^{T}(t) e(t) .
\end{gathered}
$$

Then, it follows from (3.6) and $\Psi_{1}:=\varepsilon_{2}^{1 / 2} e^{T}(t) P-\varepsilon_{2}^{-1 / 2} l^{T}(t), \Psi_{1} \Psi_{1}^{T} \geq 0$ that

$$
e^{T}(t) P l(t)+l^{T}(t) P e(t) \leq e^{T}(t)\left(\varepsilon_{2} P^{2}+\varepsilon_{2}^{-1} a_{11}^{2} I\right) e(t) .
$$

Next, it results from (3.7) and $\Psi_{2}:=\varepsilon_{3}^{1 / 2} e^{T}(t) P-\varepsilon_{3}^{-1 / 2} m^{T}(t-\tau), \Psi_{2} \Psi_{2}^{T} \geq 0$ that

$$
e^{T}(t) P m(t-\tau)+m^{T}(t-\tau) P e(t) \leq \varepsilon_{3} e^{T}(t) P^{2} e(t)+\varepsilon_{3}^{-1} a_{22}^{2} e^{T}(t-\tau) e(t-\tau) .
$$

Similarly, from (3.8) and $\Psi_{3}:=\varepsilon_{4}^{1 / 2} e^{T}(t) P K+\varepsilon_{4}^{-1 / 2} n^{T}(t), \Psi_{3} \Psi_{3}^{T} \geq 0$, we have

$$
-e^{T}(t) P K n(t)-n^{T}(t) K^{T} P e(t) \leq \varepsilon_{4} e^{T}(t)\left(P K K^{T} P\right) e(t)+\varepsilon_{4}^{-1} a_{33}^{2} e^{T}(t) e(t) .
$$

For simplicity, we denote

$$
\begin{gathered}
\Pi:=(A-K C)^{T} P+P(A-K C)+P\left[\left(\varepsilon_{1}+\varepsilon_{2}+\varepsilon_{3}\right) I+\varepsilon_{4} K K^{T}\right] P \\
+\left(\varepsilon_{2}^{-1} a_{11}^{2}+\varepsilon_{4}^{-1} a_{33}^{2}\right) I+\varepsilon_{1}^{-1} A_{d}^{T} A_{d}+\varepsilon_{3}^{-1} a_{22}^{2} I
\end{gathered}
$$

and then (3.1)(3.2) indicate that $\Pi<0$.

Denote

$$
\zeta:=\operatorname{tr}\left[\left(E_{1}-K E_{2}\right)\left(E_{1}-K E_{2}\right)^{T} P\right] .
$$

Substituting (3.2), (3.5) and (3.9)-(3.11) into (3.4) results in

$$
\begin{aligned}
\frac{d}{d t} V(e(t), t) & \leq e^{T}(t) \Pi e(t)+\zeta+2 e^{T}(t) P\left(E_{1}-K E_{2}\right) w(t) \\
& \leq-\lambda_{\min }(-\Pi)|e(t)|^{2} d t+\zeta+2 e^{T}(t) P\left(E_{1}-K E_{2}\right) w(t) .
\end{aligned}
$$

To show the expected exponential ultimate boundedness (in the mean square) of solution to the system (2.12), we shall make some standard manipulations on the relation (3.14) by utilizing the technique developed in $[8,9]$.

Let $\beta>0$ be the unique root of the equation

$$
\lambda_{\min }(-\Pi)-\beta \lambda_{\max }(P)-\beta \tau \lambda_{\max }(Q) e^{\beta \tau}=0
$$

where $\Pi$ and $Q$ are defined, respectively, in (3.12) and (3.2), $P$ is the positive definite solution to (3.1), and $\tau$ is the time-delay.

We can obtain from (3.14) that

$$
\begin{aligned}
d\left[e^{\beta t} V(e(t), t)\right]= & e^{\beta t}[\beta V(e(t), t) d t+d V(e(t), t)] \\
\leq & e^{\beta t}\left(-\left[\lambda_{\min }(-\Pi)-\beta \lambda_{\max }(P)\right]|e(t)|^{2}+\beta \lambda_{\max }(Q) \int_{t-\tau}^{t}|e(s)|^{2} d s\right) d t \\
& +\zeta e^{\beta t} d t+2 e^{\beta t} e^{T}(t) P\left(E_{1}-K E_{2}\right) w(t) d t .
\end{aligned}
$$


Then, integrating both sides from 0 to $T>0$ and taking the expectation result in

$$
\begin{aligned}
e^{\beta T} \mathbb{E} V(e(T), T) \leq & {\left[\lambda_{\max }(P)+\tau \lambda_{\max }(Q)\right] \sup _{-\tau \leq \theta \leq 0} \mathbb{E}|\xi(\theta)|^{2} } \\
& -\left[\lambda_{\min }(-\Pi)-\beta \lambda_{\max }(P)\right] \mathbb{E} \int_{0}^{T} e^{\beta t}|e(t)|^{2} d t \\
& +\beta \lambda_{\max }(Q) \mathbb{E} \int_{0}^{T} e^{\beta t} \int_{t-\tau}^{t}|e(s)|^{2} d s d t+\zeta \beta^{-1}\left(e^{\beta T}-1\right) .
\end{aligned}
$$

Note that

$$
\begin{aligned}
& \int_{0}^{T} e^{\beta t} \int_{t-\tau}^{t}|e(s)|^{2} d s d t \leq \int_{-\tau}^{T}\left(\int_{\max (s, 0)}^{\min (s+\tau, T)} e^{\beta t} d t\right)|e(s)|^{2} d s \\
& \leq \int_{-\tau}^{T} \tau e^{\beta(s+\tau)}|e(s)|^{2} d s \leq \tau e^{\beta \tau} \int_{0}^{T} e^{\beta t}|e(t)|^{2} d t+\tau e^{\beta \tau} \int_{-\tau}^{0}|\xi(\theta)|^{2} d \theta .
\end{aligned}
$$

Then, considering the definition of $\beta$ in (3.15), we have

$$
\begin{aligned}
e^{\beta T} \mathbb{E} V(e(T), T) \leq & {\left[\lambda_{\max }(P)+\tau \lambda_{\max }(Q)\right] \sup _{-\tau \leq \theta \leq 0} \mathbb{E}|\xi(\theta)|^{2} } \\
& +\beta \lambda_{\max }(Q) \tau^{2} e^{\beta \tau} \sup _{-\tau \leq \theta \leq 0} \mathbb{E}|\xi(\theta)|^{2}+\zeta \beta^{-1}\left(e^{\beta T}-1\right),
\end{aligned}
$$

and

$$
\begin{aligned}
\mathbb{E}|e(T)|^{2} \leq & \lambda_{\min }^{-1}(P)\left(\left[\lambda_{\max }(P)+\tau \lambda_{\max }(Q)\right] \sup _{-\tau \leq \theta \leq 0} \mathbb{E}|\xi(\theta)|^{2}\right. \\
& \left.+\beta \lambda_{\max }(Q) \tau^{2} e^{\beta \tau} \sup _{-\tau \leq \theta \leq 0} \mathbb{E}|\xi(\theta)|^{2}\right) e^{-\beta T}+\zeta \beta^{-1} \lambda_{\min }^{-1}(P)\left(e^{\beta T}-1\right) e^{-\beta T} .
\end{aligned}
$$

Notice that $\left(e^{\beta T}-1\right) e^{-\beta T}<1$ and let

$$
\alpha:=\lambda_{\min }^{-1}(P)\left[\lambda_{\max }(P)+\tau \lambda_{\max }(Q)\left(1+\beta \tau e^{\beta \tau}\right)\right], \quad \gamma:=\zeta \beta^{-1} \lambda_{\min }^{-1}(P) .
$$

Since $T>0$ is arbitrary, the definition of exponential ultimate boundedness in (2.13) is then satisfied, and this completes the proof of Theorem 3.1.

\subsection{Filter design}

In this subsection we shall focus on deriving the explicit expression of expected filter gains by using the algebraic matrix inequalities. As indicated in the introduction, in most literature concerning nonlinear filtering, the solution to the nonlinear filtering problem has not been given as an explicit representation.

Based on Theorem 3.1, our design problem is converted into the following equivalent "assignment problem":

- For the matrix inequality (3.1), find the necessary and sufficient conditions for the positive definite matrix $P$ under which there exists a filter gain $K$ satisfying (3.1).

- If the filter gain $K$ exists, give the characterization of all expected filter gains in terms of the positive definite matrix $P$ and some other free parameters. 
Prior to introducing our main results, we first give the following important lemma.

Lemma 3.1 [4] Let $X \in \mathbb{R}^{m_{1} \times n_{1}}$ and $Y \in \mathbb{R}^{m_{1} \times p_{1}}\left(m_{1} \leq p_{1}\right)$. There exists a matrix $U \in$ $\mathbb{R}^{n_{1} \times p_{1}}$ which simultaneously satisfies $Y=X U$ and $U U^{T}=I$ if and only if $X X^{T}=Y Y^{T}$.

For presentation convenience, we define

$$
\begin{aligned}
\Gamma\left(\varepsilon_{1}, \varepsilon_{2}, \varepsilon_{3}, \varepsilon_{4}, P\right):= & A^{T} P+P A+\left(\varepsilon_{1}+\varepsilon_{2}+\varepsilon_{3}\right) P^{2} \\
& +\left(\varepsilon_{2}^{-1} a_{11}^{2}+\varepsilon_{4}^{-1} a_{33}^{2}\right) I+Q, \\
\Xi\left(\varepsilon_{1}, \varepsilon_{2}, \varepsilon_{3}, \varepsilon_{4}, P\right):= & A^{T} P+P A+\left(\varepsilon_{1}+\varepsilon_{2}+\varepsilon_{3}\right) P^{2}+\varepsilon_{1}^{-1} A_{d}^{T} A_{d} \\
& +\left(\varepsilon_{2}^{-1} a_{11}^{2}+\varepsilon_{3}^{-1} a_{22}^{2}+\varepsilon_{4}^{-1} a_{33}^{2}\right) I-\varepsilon_{4}^{-1} C^{T} C,
\end{aligned}
$$

where $Q$ is defined in (3.2).

The following theorem solves the aforementioned assignment problem.

Theorem 3.2 There exist positive scalars $\varepsilon_{1}, \varepsilon_{2}, \varepsilon_{3}, \varepsilon_{4}$ and a positive definite matrix $P$ such that the matrix inequality (3.1) has a solution $K$ if and only if the following quadratic matrix inequality

$$
\begin{gathered}
\Xi\left(\varepsilon_{1}, \varepsilon_{2}, \varepsilon_{3}, \varepsilon_{4}, P\right)=A^{T} P+P A+\left(\varepsilon_{1}+\varepsilon_{2}+\varepsilon_{3}\right) P^{2}+\varepsilon_{1}^{-1} A_{d}^{T} A_{d} \\
+\left(\varepsilon_{2}^{-1} a_{11}^{2}+\varepsilon_{3}^{-1} a_{22}^{2}+\varepsilon_{4}^{-1} a_{33}^{2}\right) I-\varepsilon_{4}^{-1} C^{T} C<0
\end{gathered}
$$

holds. Furthermore, if (3.18) is true, all matrices $K$ meeting the matrix inequality (3.1) can be parameterized by

$$
K=\varepsilon_{4}^{-1} P^{-1} C^{T}+\varepsilon_{4}^{-1 / 2} P^{-1} \Lambda U
$$

where $\Lambda \in \mathbb{R}^{n \times p}$ is any matrix satisfying

$$
\Lambda \Lambda^{T}<-\Xi\left(\varepsilon_{1}, \varepsilon_{2}, \varepsilon_{3}, \varepsilon_{4}, P\right)
$$

and $U \in \mathbb{R}^{p \times p}$ is arbitrary orthogonal matrix (i.e., $U U^{T}=I$ ).

Proof: It is easy to rewrite the matrix inequality (3.1) as

$$
\begin{gathered}
-C^{T} K^{T} P-P K C+\varepsilon_{4} P K K^{T} P+A^{T} P+P A \\
+\left(\varepsilon_{1}+\varepsilon_{2}+\varepsilon_{3}\right) P^{2}+\left(\varepsilon_{2}^{-1} a_{11}^{2}+\varepsilon_{4}^{-1} a_{33}^{2}\right) I+Q<0,
\end{gathered}
$$

or by the definition (3.16),

$$
-C^{T} K^{T} P-P K C+\varepsilon_{4} P K K^{T} P+\Gamma\left(\varepsilon_{1}, \varepsilon_{2}, \varepsilon_{3}, \varepsilon_{4}, P\right)<0 .
$$

We continue to rearrange $(3.22)$ as

$$
\begin{gathered}
{\left[\varepsilon_{4}^{1 / 2} P K-\varepsilon_{4}^{-1 / 2} C^{T}\right]\left[\varepsilon_{4}^{1 / 2} P K-\varepsilon_{4}^{-1 / 2} C^{T}\right]^{T}} \\
<\varepsilon_{4}^{-1} C^{T} C-\Gamma\left(\varepsilon_{1}, \varepsilon_{2}, \varepsilon_{3}, \varepsilon_{4}, P\right) .
\end{gathered}
$$

Obviously, there exists a filter gain matrix $K$ such that the inequality (3.1) (or equivalently (3.23)) holds for some positive scalars $\varepsilon_{1}, \varepsilon_{2}, \varepsilon_{3}, \varepsilon_{4}$ and positive definite matrix $P$ if and 
only if the right-hand side of (3.23) is positive definite, i.e., $-\Xi\left(\varepsilon_{1}, \varepsilon_{2}, \varepsilon_{3}, \varepsilon_{4}, P\right)>0$ or (3.18) holds. This proves the first part of this theorem.

We now assume that (3.18) is true. Note that the dimension of the filter gain $K$ is $n \times p$ and $p \leq n$. From (3.23) and the definition of $\Lambda \in \mathbb{R}^{n \times p}$ in (3.20), we have

$$
\left[\varepsilon_{4}^{1 / 2} P K-\varepsilon_{4}^{-1 / 2} C^{T}\right]\left[\varepsilon_{4}^{1 / 2} P K-\varepsilon_{4}^{-1 / 2} C^{T}\right]^{T}=\Lambda \Lambda^{T} .
$$

It then follows from Lemma 3.1 that (3.24) holds if and only if

$$
\varepsilon_{4}^{1 / 2} P K-\varepsilon_{4}^{-1 / 2} C^{T}=\Lambda U
$$

where $U \in \mathbb{R}^{p \times p}$ is an arbitrary orthogonal matrix. Therefore, the expression (3.19) follows immediately. This completes the proof of the theorem.

Finally, we sum up our results in the following theorem that is easily accessible from Theorem 3.1 and Theorem 3.2.

Theorem 3.3 Consider the nonlinear continuous-time state delayed stochastic system (2.1)-(2.3) and the associated nonlinear filter (2.7). If there exist positive scalars $\varepsilon_{1}, \varepsilon_{2}, \varepsilon_{3}, \varepsilon_{4}$ and a positive definite matrix $P$ such that the matrix inequality (3.18) holds, then the filter (2.7) with its parameter given in (3.19) will be such that the dynamics of the estimation error (i.e., the solution of the error-state system (2.12)) is stochastically exponentially ultimately bounded in the mean square.

Remark 3.1 Theorem 3.3 gives the solution to the addressed filter design problem for nonlinear time-delay stochastic systems in this paper. In practical applications, it is very desirable to directly solve the quadratic matrix inequality (3.18), and then obtain the expected filter parameters from (3.19) easily. Thus, necessary discussion on the numerical algorithm is in order now. Firstly, based on the algorithms provided in [18], we may select appropriate positive scalar parameters $\varepsilon_{1}, \varepsilon_{2}, \varepsilon_{3}, \varepsilon_{4}$ so as to minimize (or reduce) the conservativeness resulting from the utilization of the inequalities (3.5) and (3.9)-(3.11). Then, (3.18) will be a standard quadratic matrix inequality (QMI) for $P$. For details concerning the general QMIs and relevant algorithms, we refer the reader to [13]. Specifically, we now briefly discuss the conditions for the existence of a positive definite solution to the QMI (3.18). Assume that

$$
\Gamma_{1}:=\varepsilon_{1}^{-1} A_{d}^{T} A_{d}+\left(\varepsilon_{2}^{-1} a_{11}^{2}+\varepsilon_{3}^{-1} a_{22}^{2}+\varepsilon_{4}^{-1} a_{33}^{2}\right) I-\varepsilon_{4}^{-1} C^{T} C>0 .
$$

It is easily accessible from [7] that, there exists a positive definite solution $P$ to the QMI (3.18) if and only if

$$
\left\|\Gamma_{1}^{1 / 2}(s I-A)^{-1}\left(\varepsilon_{1}+\varepsilon_{2}+\varepsilon_{3}\right)^{1 / 2} I\right\|_{\infty}<1
$$

where $\|H(s)\|_{\infty}=\sup _{\omega \in \mathbb{R}} \sigma_{\max }[H(j \omega)]$ and $\sigma_{\max }[\cdot]$ is the largest singular value of [·].

Remark 3.2 It is clear that, if the set of desired filter gains is not empty, it must be very large. We may utilize the freedom (such as the choices of matrices $\Lambda$ and $U$ ) in the filter design to improve other system properties. An interesting problem for future research is how to exploit such freedom to achieve the reliable constraint on the filtering process. 


\section{Some extensions}

\subsection{Case 1}

Let us first deal with the multiple state delay case. Consider the following nonlinear continuous-time multi-delay stochastic system

$$
\dot{x}(t)=f(x(t), u(t))+\sum_{i=1}^{r} g_{i}\left(x\left(t-\tau_{i}\right)\right)+E_{1} w(t), \quad x(t)=\varphi(t), \quad t \in[-\tau, 0],
$$

together with same measurement equation as $(2.3)$. The nonlinear vector functions $f(\cdot, \cdot)$ and $h(\cdot)$ satisfy the same constraints as in $(2.4)$ and (2.6) respectively, while the nonlinear vector functions $g_{i}(\cdot)(i=1,2, \cdots, r)$ meet $g_{i}(0)=0$ and

$$
\left|g_{i}\left(x\left(t-\tau_{i}\right)+\sigma_{i}\right)-g_{i}\left(x\left(t-\tau_{i}\right)\right)-A_{d i} \sigma_{i}\right| \leq a_{22 i}\left|\sigma_{i}\right|
$$

where $A_{d i} \in \mathbb{R}^{n \times n}$ is a known constant matrix, $\sigma_{i} \in \mathbb{R}^{n}$ is a vector and $a_{22 i}$ is a known positive constant $(1 \leq i \leq r)$.

We adopt the following filter structure

$$
\dot{\hat{x}}(t)=f(\hat{x}(t), u(t))+\sum_{i=1}^{r} g_{i}\left(\hat{x}\left(t-\tau_{i}\right)\right)+K[y(t)-h(\hat{x}(t))]
$$

and we may obtain an error-state system which is similar to (2.12). Then, instead of (3.3), we define the Lyapunov functional $V(e(t), t)=e^{T}(t) P e(t)+\sum_{i=1}^{r} \int_{t-\tau_{i}}^{t} e^{T}(s) Q_{i} e(s) d s$. By using the same idea exploited in the proof of Theorem 3.1 and Theorem 3.2, we can easily obtain the parallel results for the multi-delay case.

\subsection{Case 2}

We now cope with the case where there are bounded nonlinearities and uncertain disturbances. In this case, the system under consideration is the same as (2.1)-(2.3). However, the constraints (2.4)-(2.6) are replaced by (see [21]):

$$
\begin{gathered}
\left|f(x(t)+\sigma, u(t)+\delta)-f(x(t), u(t))-\left[\begin{array}{ll}
A & B
\end{array}\right]\left[\begin{array}{l}
\sigma \\
\delta
\end{array}\right]\right| \leq a_{11}\left|\left[\begin{array}{l}
\sigma \\
\delta
\end{array}\right]\right|+b_{11} \\
\left|g(x(t-\tau)+\sigma)-g(x(t-\tau))-A_{d} \sigma\right| \leq a_{22}|\sigma|+b_{22} \\
|h(x(t)+\sigma)-h(x(t))-C \sigma| \leq a_{33}|\sigma|+b_{33}
\end{gathered}
$$

where the extra constants $b_{11}, b_{22}$ and $b_{33}$ are used to reflect the possible bounded nonlinearities and disturbances of the uncertain forms.

Consider the system (2.1)-(2.3) with the constraints (4.4)-(4.6). We now follow the same line of the proof of Theorem 3.1. In the light of the simple algebraic inequality $(x+y)^{2} \leq$ $2 x^{2}+2 y^{2}$ where $x$ and $y$ are real scalars, we can replace the inequalities (3.6)-(3.8) by the followings:

$$
\begin{gathered}
l^{T}(t) l(t)=|f(x(t), u(t))-f(\hat{x}(t), u(t))-A e(t)|^{2} \leq 2 a_{11}^{2} e^{T}(t) e(t)+2 b_{11}^{2}, \\
m^{T}(t-\tau) m(t-\tau)=\left|g(x(t-\tau))-g(\hat{x}(t-\tau))-A_{d} e(t-\tau)\right|^{2} \\
\leq 2 a_{22}^{2} e^{T}(t-\tau) e(t-\tau)+2 b_{22}^{2} \\
n^{T}(t) n(t)=|h(x(t))-h(\hat{x}(t))-C e(t)|^{2} \leq 2 a_{33}^{2} e^{T}(t) e(t)+2 b_{33}^{2} .
\end{gathered}
$$


Then, similar to the proof of Theorem 3.1, the stochastic derivative of $V$ (see (3.3)) along a given trajectory satisfies

$$
\begin{aligned}
\frac{d}{d t} V(e(t), t) \leq & -\lambda_{\min }\left(-\Pi_{1}\right)|e(t)|^{2} d t+\zeta+\left(2 b_{11}^{2}+2 b_{22}^{2}+2 b_{33}^{2}\right) \\
& +2 e^{T} P\left(E_{1}-K E_{2}\right) w(t)
\end{aligned}
$$

where

$$
\begin{gathered}
\Pi_{1}:=(A-K C)^{T} P+P(A-K C)+P\left[\left(\varepsilon_{1}+\varepsilon_{2}+\varepsilon_{3}\right) I+\varepsilon_{4} K K^{T}\right] P \\
+\left(2 \varepsilon_{2}^{-1} a_{11}^{2}+2 \varepsilon_{4}^{-1} a_{33}^{2}\right) I+\varepsilon_{1}^{-1} A_{d}^{T} A_{d}+2 \varepsilon_{3}^{-1} a_{22}^{2} I .
\end{gathered}
$$

Now, along the line of the proof of Theorem 3.1 in the previous section, we arrive at the following result easily.

Theorem 4.1 Consider the system (2.1)-(2.3) with the constraints (4.4)-(4.6). Let the filter parameter $K$ be given. If there exist positive scalars $\varepsilon_{1}, \varepsilon_{2}, \varepsilon_{3}, \varepsilon_{4}$ such that the matrix inequality $\Pi_{1}<0$ has a positive definite solution $P>0$, then the estimation error (the solution to the system (2.12)) is stochastically exponentially ultimately bounded in the mean square.

Subsequently, we can obtain analogous results for the filter design as in Theorem 3.2 and Theorem 3.3. The reason why we discuss the system (2.1)-(2.3) associated with (2.4)(2.6) is to make our theory more understandable and to avoid unnecessarily complicated notations.

\section{Numerical simulation}

Let the nonlinear stochastic state delayed system be described by

$$
\begin{aligned}
\dot{x_{1}}(t)= & -3.5 x_{1}(t)+0.1 x_{2}(t)-0.4 x_{3}(t)+0.1 \sin \left(x_{1}(t)+x_{2}(t)\right) \\
& +0.04 x_{1}(t-0.1)-0.01 x_{2}(t-0.1)-0.01 x_{3}(t-0.1)+0.2 w(t), \\
\dot{x_{2}}(t)= & -0.5 x_{1}(t)-2 x_{2}(t)+0.6 x_{3}(t)+0.2 x_{2}(t) \sin x_{2}(t) \\
& +0.01 x_{1}(t-0.1)-0.03 x_{2}(t-0.1)+0.02 x_{3}(t-0.1)+0.2 w(t), \\
\dot{x_{3}}(t)= & -0.5 x_{1}(t)+0.8 x_{2}(t)-4 x_{3}(t)+0.1 \sin \left(x_{2}(t)-x_{3}(t)\right) \\
& +0.01 x_{1}(t-0.1)-0.01 x_{2}(t-0.1)+0.05 x_{3}(t-0.1)+0.2 w(t), \\
y_{1}(t)= & x_{1}(t)+0.2 \sin x_{2}(t)+0.1 w(t) ; \\
y_{2}(t)= & x_{2}(t)+0.1 \sin x_{1}(t)+0.1 w(t) ; \\
y_{3}(t)= & x_{3}(t)+0.3 \sin x_{3}(t)+0.1 w(t) .
\end{aligned}
$$

Considering the system (2.1)-(2.3) with the constraints (2.4)-(2.6), we can obtain that

$$
\begin{gathered}
A=\left[\begin{array}{ccc}
-3.5 & 0.1 & -0.4 \\
-0.5 & -2 & 0.6 \\
-0.5 & 0.8 & -4
\end{array}\right], A_{d}=\left[\begin{array}{ccc}
0.04 & -0.01 & -0.01 \\
0.01 & -0.03 & 0.02 \\
0.01 & -0.01 & 0.05
\end{array}\right], B=0, \\
E_{1}=\left[\begin{array}{ccc}
0.2 & 0.2 & 0.2
\end{array}\right]^{T}, E_{2}=\left[\begin{array}{lll}
0.01 & 0.01 & 0.01
\end{array}\right]^{T}, C=I_{3}, \\
\tau=0.1, \quad a_{11}=0.4899, \quad a_{22}=0, \quad a_{33}=0.7483 .
\end{gathered}
$$


We want to design an exponential filter for the nonlinear time-delay system (2.1)-(2.3), such that the dynamics of the estimation error is stochastically exponentially ultimately bounded in the mean square. Firstly, the parameters $\varepsilon_{i}(i=1,2,3,4)$ are chosen as: $\varepsilon_{1}=0.2, \varepsilon_{2}=2.1, \varepsilon_{3}=0.6, \varepsilon_{4}=0.5$. Then, a positive definite solution to (3.18) and the matrix $\Xi$ are calculated respectively as follows:

$$
P=\left[\begin{array}{ccc}
2.4183 & 0.0655 & 0.2911 \\
0.0655 & 1.4131 & -0.5095 \\
0.2911 & -0.5095 & 2.7390
\end{array}\right], \quad \Xi=\left[\begin{array}{ccc}
-0.8232 & -0.0436 & 0.0325 \\
-0.0436 & -0.6585 & 0.0145 \\
0.0325 & 0.0145 & -0.7524
\end{array}\right]
$$

To show the design freedom, we now discuss two cases.

Case 1: We set $\Lambda=0.8 I_{3}, U=I_{3}$, and obtain from (3.19) the desired filter gain as

$$
K=\left[\begin{array}{ccc}
1.3176 & -0.1196 & -0.1623 \\
-0.1196 & 2.3860 & 0.4565 \\
-0.1623 & 0.4565 & 1.2454
\end{array}\right]
$$

Denote the error states $e_{i}=x_{i}-\hat{x}_{i}(i=1,2,3)$. The responses of error dynamics to initial conditions are shown in Fig. 1 (All the figures are displayed at the end of this paper), and the real state $x_{1}$ (respectively, $x_{2}, x_{3}$ ) and its estimate $\hat{x}_{1}$ (respectively, $\hat{x}_{2}, \hat{x}_{3}$ ) are displayed in Fig. 2 (respectively, Fig. 3, Fig. 4). The simulation results imply that the desired goal is well achieved.

Case 2: In this case we select $\Lambda=0.8 I_{3}, U=\operatorname{diag}\{-1,-1,1\}$, and get

$$
K=\left[\begin{array}{ccc}
0.3655 & -0.0332 & -0.1623 \\
-0.0332 & 0.6619 & 0.4565 \\
-0.0450 & 0.1266 & 1.2454
\end{array}\right]
$$

The simulation results for Case 2, which again show that our expected performance is well guaranteed, are omitted to save the space. We can see from the simulation that, the mean square exponential rate of convergence for the estimation error, which reflects the steady-state performance of the designed filter, is ensured. On the other hand, it would be more practical to improve the transient behavior for a nonlinear process. This gives us one of the future research topics.

\section{Conclusions}

In this paper we have considered the filter design problem for a class of nonlinear stochastic time-delay systems. Both the filter analysis and design issues have been discussed in detail by means of quadratic matrix inequalities. We have derived the existence conditions as well as the analytical parameterization of desired filters. The method relies not on the optimization theory but on Lyapunov type stochastic stability results which can guarantee a mean square exponential rate of convergence for the estimation error. It has been emphasized that, the desired exponential filters for this class of nonlinear time-delay systems, when they exist, are usually a large set, and the remaining freedom can be used to meet other expected performance requirements. The results of this paper have been demonstrated by a numerical simulation example. 


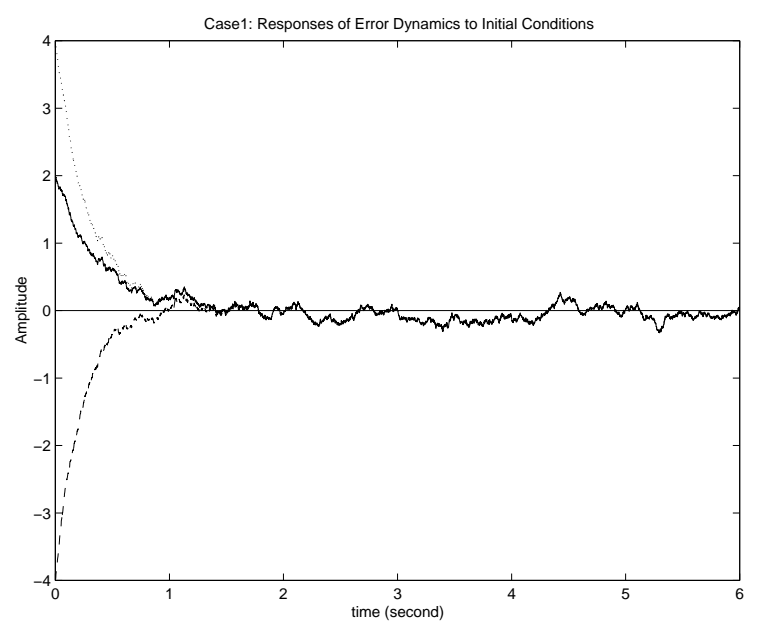

Figure 1: $e_{1}$ (solid), $e_{2}$ (point), $e_{3}$ (dashed)

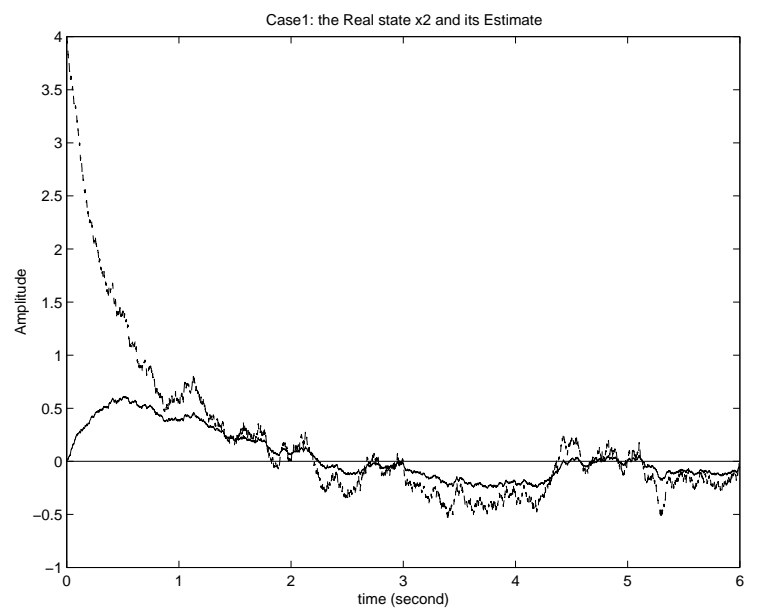

Figure 3: $x_{2}$ (dashed), $\hat{x}_{2}$ (solid).

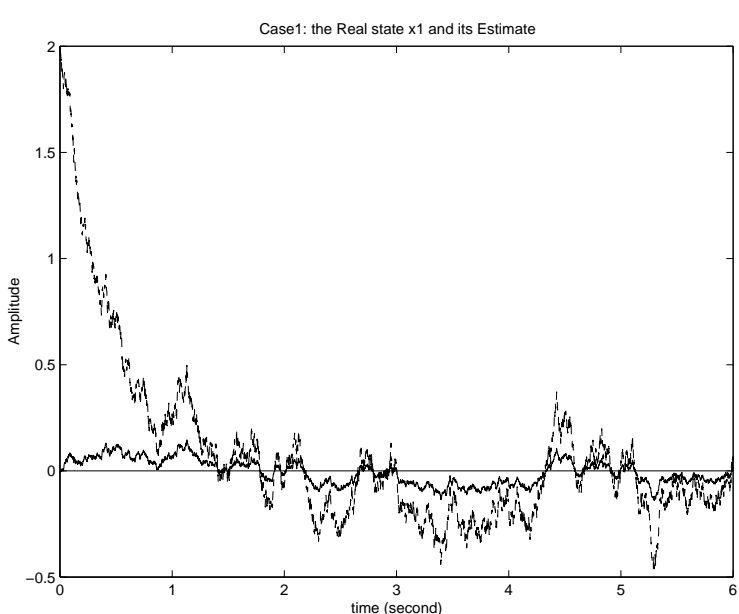

Figure 2: $x_{1}$ (dashed), $\hat{x}_{1}$ (solid).

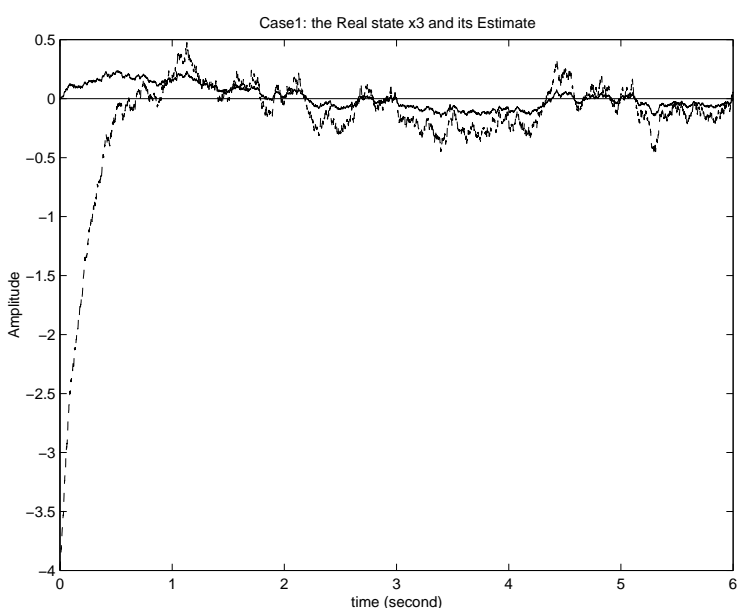

Figure 4: $x_{3}$ (dashed), $\hat{x}_{3}$ (solid).

\section{Acknowledgment}

This work of Z. Wang was supported in part by City University of Hong Kong (CityU SRG No. 7001146), University of Kaiserslautern of Germany and the Alexander von Humboldt Foundation of Germany.

\section{References}

[1] R. S. Bucy and P. D. Joseph, Filtering for stochastic processes with applications to guidance, (New York: Interscience, 1968)

[2] A. Gelb, Applied optimal estimation, (Cambridge: Cambridge University Press, 1974)

[3] F. Hsiao and S. Pan, Robust Kalman filter synthesis for uncertain multiple time-delay stochastic systems, J. Dyn. Syst. Meas. Control, vol. 118, pp. 803-808, 1996. 
[4] C. Hsieh and R. E. Skelton, All covariance controllers for linear discrete time systems. IEEE Trans. Automat. Control, vol. 35, pp. 908-915, 1990.

[5] A. H. Jazwinski, Stochastic processes and filtering theory, (New York: Academic Press, 1970)

[6] K. Ito and B. Rozovskii, Approximation of the Kushner equation for nonlinear filtering, SIAM J. Control Optimization, vol. 38, pp. 893-915, 2000.

[7] P. P. Khargonekar, I. R. Petersen and K. Zhou, Robust stabilization of uncertain linear system: quadratic stabilizability and $H_{\infty}$ control theory, IEEE Trans. Automat. Control, vol. 35, pp. 356-361, 1990.

[8] X. Mao, Robustness of exponential stability of stochastic differential delay equations, IEEE Trans. Automat. Contr., vol. 41, pp. 442-447, 1996.

[9] X. Mao, Stochastic Differential Equations and Applications, (Horwood, 1997)

[10] A. K. Mahalanabis and M. Farooq, A second-order method for state estimation of nonlinear dynamical systems, Int. J. Control, vol. 14, pp. 631-639, 1971.

[11] M. S. Mahmoud, N. F. Al-Muthairi and S. Bingulac, Robust Kalman filtering for continuous time-lag systems, Systems and Control Lett., vol. 38, pp. 309-319, 1999.

[12] S. -I. Niculescu, E. I. Verriest, L. Dugard and J. M. Dion, Stability and robust stability of time-delay systems: a guided tour, In: Stability and control of time-delay systems, L. Dugard et al. (ed.) Berlin: Springer, Lect. Notes Control Inf. Sci., vol. 228, pp. 1-71, 1998.

[13] A. Saberi, P. Sannuti and B. M. Chen, $\mathrm{H}_{2}$ optimal control, (Prentice Hall International, Series in Systems and Control Engineering, London, 1995)

[14] B. M. Scherzinger and R. H. Kwong, Estimation and control of discrete time stochastic systems having cone- bounded nonlinearities, Int. J. Control, vol. 36, pp. 33-52, 1982.

[15] T. -J. Tarn and Y. Rasis, Observers for nonlinear stochastic systems, IEEE Trans. Signal Processing, vol. 21, pp. 441-448, 1976.

[16] Z. Wang, B. Huang and H. Unbehauen, Robust $H_{\infty}$ observer design of linear state delayed systems with parametric uncertainty: the discrete-time case, Automatica, vol. 35, 1161-1167, 1999.

[17] Z. Wang, B. Huang and H. Unbehauen, Robust $\mathcal{H}_{\infty}$ observer design of linear time-delay systems with parametric uncertainty, Systems \& Control Letters, vol. 42, 303-312, 2001.

[18] L. Xie and Y. C. Soh, Robust Kalman filtering for uncertain systems, Systems and Control Lett., vol. 22, pp. 123-129, 1994.

[19] Y. Yavin, Numerical studies in nonlinear filtering, (Lecture Notes in Control and Information Sciences, vol. 65, Berlin: Springer-Verlag, 1985)

[20] E. Yaz, Linear state estimators for non-linear stochastic systems with noisy non-linear observations, Int. J. Control, vol. 48, pp. 2465-2475.

[21] E. Yaz and A. Azemi, Observer design for discrete and continuous non-linear stochastic systems, Int. J. Syst. Sci., vol. 24, pp. 2289-2302, 1993.

[22] M. Zakai, On the ultimate boundedness of moments associated with solutions of stochastic differential equations, SIAM J. Control, vol. 5, pp. 588-593, 1967. 